\title{
"Non avere paura": prevenire le discriminazioni attraverso l'educazione interculturale*
}

\author{
di Marco Catarci^, Massimiliano Fiorucci, Maria Chiara Giorda ${ }^{\circ}$, \\ Gennaro Gervasio^, Manfredi Merluzzi^, Paola Perucchini^
}

\section{Riassunto}

Il contributo che proponiamo offre una riflessione sulla confusione semantica e sui limiti dei discorsi sulla radicalizzazione che spesso contribuiscono ad alimentare stereotipi e pregiudizi, accelerando la diffusione di paure sociali già storicamente radicate, come quelle relative alle diversità culturali e religiose. Anziché soffermarci su modi e tecniche di azioni preventive di processi (almeno mediaticamente) sopravvalutati, proponiamo che l'educazione interculturale sia l'approccio più fruttuoso per operare una pulizia lessicale e concettuale, per prevenire le discriminazioni - in primis l'islamofobia - come mostrano i risultati del progetto PriMed-Prevenzione e interazione nello spazio trans-mediterraneo, per gettare le basi di legami sociali e culturali complessi e plurali. La prospettiva interdisciplinare si fonda su differenti approcci metodologici e su una variegata e recente letteratura proveniente, in particolare, dall'ambito pedagogico, psicologico, sociologico e storico.

Parole chiave: fondamentalismi religiosi; radicalizzazione; educazione interculturale; Islam.

\footnotetext{
* Il contributo rientra tra le attività di ricerca del progetto PriMED (https://primed-miur.it); si tratta di un progetto di prevenzione della radicalizzazione religiosa. Lanciato dal Ministero dell'Istruzione, dell'università e della ricerca scientifica mediante il bando MIUR: "Costituzione di reti universitarie italiane in attuazione di accordi di cooperazione tra le università italiane e quelle di Stati aderenti all'Organizzazione della cooperazione islamica" (Decreto Direttoriale n. 3089 del 16/11/2018 - ID 82382), di durata triennale, è stato vinto da un'ampia cordata di 12 università italiane e 10 università appartenenti ai paesi OCI- Organizzazione della Conferenza (coordinata dall'Università del Piemonte Orientale) e del progetto "Dinamiche pubbliche della paura e cittadinanza inclusiva" (Azione 4: azione sperimentale di finanziamento a progetti di ricerca innovativi e di natura interdisciplinare), finanziato dall'Università degli Studi Roma Tre.

${ }^{\wedge}$ Università degli studi Roma Tre.

○Università degli studi Roma Tre. Corresponding author: mariachiara.giorda@uniroma3.it.
}

Educational Reflective Practices (ISSNe 2279-9605), 1/2021 Special Issue

Doi: 10.3280/erp1-special-2021oa12465 


\title{
"Don't be afraid": Prevent discriminations and radicalism through intercultural education
}

\begin{abstract}
The contribution we propose offers a reflection on the semantic confusion and limitations of discourses on radicalization that often contribute to feed stereotypes and prejudices, accelerating the spread of social fears already historically rooted, such as those related to cultural and religious diversity. Instead of dwelling on ways and techniques of preventive actions of processes (at least in the media) overrated, we propose that intercultural education is the most fruitful approach to operate a lexical and conceptual cleaning, to prevent discrimination - primarily Islamophobia - as shown by the results of the project PriMed-Prevention and Interaction in the TransMediterranean space, to lay the foundations of complex and plural social and cultural ties. The interdisciplinary perspective is based on different methodological approaches and on a varied and recent literature coming, in particular, from the pedagogical, psychological, sociological and historical fields.
\end{abstract}

Keywords: religious fundamentalisms; radicalization; intercultural education; Islam.

First submission: 16/07/2021, accepted: 13/09/2021

Available online: 30/09/2021

\section{Fondamentalismi, radicalismi, radicalizzazione}

Il dibattito (anche scientifico) sui termini fondamentalismo, radicalismo e radicalizzazione rischia costantemente di essere acceso da una miscela fatta di confusioni lessicali e concettuali, di un'ampia gamma di interpretazioni e di una serie di ambigue operazioni di interscambio. La storia degli studi dei singoli termini è recente e accidentata da ostacoli epistemologici e interpretativi.

Negli ultimi cinquant'anni circa, i fondamentalismi religiosi sono stati comparati e osservati da prospettive differenti (per una sintesi, Giorda, 2012): dal pionieristico "Fundamentalism project", studiosi di variegati campi disciplinari hanno messo in luce caratteristiche, strumenti, obiettivi e relazioni interne ed esterne, moltiplicando la produzione critica soprattutto a partire dal 2001 e arrivando anche a ispirare il cinema e la televisione: basti pensare al successo di un documentario come "Jesus Camp" realizzato dalle 
registe Rachel Gradi e Heidi Ewing nel 2006 che narra le dinamiche e le relazioni della "Kids on Fire school of Ministry", campo estivo che si trova sul Devil's Lake in Nord Dakota, gestito dalla pastora Becky Fischer o della mini serie trasmessa su Netflix intitolata "Unorthodox", che narra la vicenda di una giovane donna ebrea chassidica che fugge da Brooklyn a Berlino creata da Anna Winger e Alexa Karolinski Winger \& Karolinski, nel 2020, basata sull'autobiografia del 2012 di Deborah Feldman.

In Italia, dopo il lavoro pionieristico sui Fondamentalismi curato da $\mathrm{Pa}-$ squinelli (1993) resta senza dubbio un riferimento importante il lavoro dei sociologi Guolo e Pace, I fondamentalismi del 1998, più volte ripreso da altri studiosi, rielaborato, aggiornato e arricchito di casi di studio. Dal nostro punto di vista esso ha il pregio di aver messo in luce la natura ibrida e la labilità dei confini di qualsiasi definizione che abbia tentato, invano, di incatenare e cristallizzare fenomeni dinamici e in continuo cambiamento. I fondamentalismi religiosi infatti sono cangianti, anche in base al contesto storico, culturale e sociologico in cui essi prendono forma, che si tratti di protestanti neo-evangelici, di gruppi afferenti ai Fratelli Musulmani o di ebrei ultraortodossi.

Nell'ultimo decennio, probabilmente in associazione agli sviluppi dell'Isis/Isil/Daesh (e alla stagione delle stragi legate in gran parte a un certo tipo di violenza di matrice jihadista), il termine "fondamentalismo" ha ceduto il posto a quello di "radicalismo" - termine ancora più ambiguo e politicamente segnato - che, soprattutto nei media, ha finito per diventare il sinonimo di ogni fenomeno di estremismo e infine di terrorismo. Anche l'opzione lessicale tra radicalismo e radicalizzazione non è sempre chiara né tantomeno condivisa. Se il radicalismo è piuttosto l'esito, lo stato terminale del consolidarsi di una relazione con la violenza, la radicalizzazione è un processo. Secondo Guolo e Pace (1998, pp. 41-55) la rimozione dell'Islam come elemento fondativo degli Stati-nazione (e la loro utopia), la diffusione di ideologie politiche occidentali e il dilagare della modernità sono stati elementi scatenanti del "radicalismo" islamico. Più di recente, come ha affermato Guolo (2019) convinto che una spinta l'abbia data anche l'egemonia delle scienze sociali in ambiente anglosassone, "Il termine radicalizzazione si è progressivamente imposto nel linguaggio scientifico, sostituendo, di fatto, quello di fondamentalismo, in auge negli ultimi decenni del Novecento" (p. 38). Il carattere processuale della radicalizzazione (violenta) aiuta sia nell'analisi teorica, sia anche nell'osservazione empirica e nelle operazioni di prevenzione.

Anzitutto è utile riflettere su come, mentre il fondamentalismo è riferito a un gruppo, una collettività, l'enclave che si chiude e struttura (Squarcini \& Tavarnesi, 2007), la radicalizzazione è un fenomeno quasi sempre 
individuale: è la persona che si radicalizza e compie una traiettoria in cui è possibile ripercorrere la recrudescenza di alcune attitudini mosse da un sentimento di esclusione, umiliazione, frustrazione, ghettizzazione e rabbia. Come ha sapientemente spiegato Khoshrokovar studiando le carceri, i foreign fighters e i giovani cosiddetti "delle banlieues" (Khoshrokovar, 2002; 2014 ; 2016; 2021), la radicalizzazione passa attraverso diverse fasi: la fase di pre-radicalizzazione; 1'identificazione con i movimenti radicali; l'indottrinamento; il coinvolgimento diretto in azioni violente, in alcuni casi anche in gruppo.

Guolo (2019) ha ricordato come:

Il termine radicalizzazione descrive il processo mediante il quale un individuo, o un gruppo, mette in atto forme violente d'azione legate a un'ideologia estremista, di contenuto politico, sociale o religioso (Borum, 2001; Wilner \& Dubouloz, 2010), che si propone di combattere e rovesciare l'ordine politico e culturale dominante. Perché si possa parlare di radicalizzazione è, dunque, necessario che l'azione violenta si accompagni a un'ideologia, intesa come «concezione totale del mondo», che la legittima (p. 37).

Nato nell'ambito dei Radicalisation and Terrorism Studies (Antonelli, 2021, p. 18 e seguenti), il dibattito scientifico è stato spesso mosso, quando non influenzato, dalle esigenze istituzionali e pratiche della lotta per la prevenzione e contro la violenza e ciò non ha fatto altro che moltiplicare i limiti e le insidie delle ricerche, sia da un punto di vista metodologico, sia anche ermeneutico. Come suggerisce Antonelli (2021): "La radicalizzazione, così letta, è dunque un processo bifronte, caratterizzato da un forte grado di ambiguità ideologica negli assunti di base: è infatti un programma di ricerca fortemente orientato all'ottimizzazione scientifica del controllo sociale, come ricaduta implicita, che porta con sé l'inevitabile stigmatizzazione di tutto ciò che non è "moderato" all'interno del discorso dominante anche se non ha nulla a che fare con il terrorismo o l'accettazione della violenza politica" (p. 43).

Il problema delle continue torsioni semantiche e dell'uso distorto dei concetti - dettato da analfabetismo o da un uso strumentale - resta dunque vivo ed è acuito da un uso spesso forsennato e acritico che ne fanno i media: anche volendo riferirci al solo panorama italiano, gli esempi sarebbero innumerevoli. Facciamo nostri i quesiti di Antonelli (2021), che si domanda: "se il radicale, storicamente, può essere o non essere a favore della violenza come metodo di lotta politica e guarda alla radice dei problemi sociali per estirparli definitivamente, per quale motivo questo termine e il connesso radicalizzazione (cui possiamo aggiungere anche radicalismo, n.d.a.) oggi viene 
prevalentemente usato per riferirsi, in ultima istanza, al terrorismo e ai terroristi?" (p. 11).

\section{Molteplicità delle forme e differenti gradi di radicalizzazione: il caso italiano}

Nell'impossibilità di una definitiva pulizia e chiarezza concettuale, ci limiteremo a invocare una sorveglianza lessicale e individueremo l'esistenza di processi personali e collettivi di innescamento di violenze fisiche (ma anche psicologiche) ispirate da un certo modo di intendere una certa cultura religiosa o movimento o corrente. Questo è utile a delimitare il campo e a distinguere tale complesso fenomeno da altri fenomeni di violenza contro se stessi o altri, ispirati da ideologie politiche o altre forme di appartenenza. Ridotto in questi termini, il grado zero che allo studioso interessa affrontare è la verifica della sua esistenza, della sua percezione e delle sue ricadute, prima di ogni altra interpretazione, prima di domandarsi con Kepel e Roy se si tratti di radicalizzazione dell'Islam o di "islamizzazione della radicalità" (Guolo, 2019). Ed è proprio riferendosi al contesto italiano che sorge un altro ordine di problemi, vale a dire lo iato tra il percepito e il vissuto, tra le questioni e le esigenze che sono sollevate top-down dalle politiche di sicurezza (e securitarie) e le urgenze reali che sono espresse in una prospettiva bottomup e che minacciano la possibile convivenza tra diversità. Distorsioni, processi di stereotipizzazione, mistificazioni, e soprattutto sopravvalutazioni sono alcune delle parole chiave che permettono di leggere tale iato. La questione cruciale è quindi: in Italia quanto è un fenomeno sociale diffuso e pericoloso la radicalizzazione violenta di stampo religioso e in particolare islamico, di individui singoli o di gruppi?

Condividiamo la tesi della specificità italiana, più ancora che del "ritardo" (categoria che si limita a rimandare, dilatare nel tempo) in riferimento al radicalismo/fondamentalismo di matrice islamica, proposta da Berardinelli e Guglielminetti (2018): «In recent years Italy has not suffered jihadist terrorist attacks, with the exception of the failed attack of a suicide bomber, Libyan immigrant Mohammed Game, who attempted to blow himself up in front of the "Santa Barbara" barracks in Milan: the place from which Italian soldiers had left for ISAF missions in Afghanistan. Shouting: "Out of Afghanistan", the 35-year-old Libyan engineer suffered severe injuries to his hand and eyes, and slightly wounded the young soldier guarding the barracks» (p. 28).

L'Italia è uno degli stati a più bassa intensità di episodi di violenza legati alla religione, in particolare all'Islam: la cifra di 129 foreign fighters italiani alla fine del 2017, secondo i dati del Ministero dell'Interno, sembra 
confermare questa tendenza (Guolo, 2019; Marone \& Vidino, 2018), nonostante il clamore mediatico destato, ad esempio, dal caso di Maria Giulia "Fatima" Sergio, combattente e "arruolatrice" per conto dell'ISIS, condannata in contumacia nel 2016, dopo essere scomparsa in Siria (Serafini, 2015).

Tra le ragioni che Guolo (2019) individua per spiegare questa specificità italiana, vi sarebbe la stratificazione del ciclo delle età dovuto a un più recente fenomeno migratorio, la geografia migratoria, con l'assenza di periferie o sobborghi-ghetto, l'assenza di poli etnicizzati e in potenza radicalizzati, poiché anche i casi di Ravenna e Latina non hanno prodotto alcun fenomeno paragonabile ad agglomerati a rischio o esplosi come in altri paesi europei. Anche la caotica e mal gestita super-diversità migratoria, culturale e religiosa e la sua conseguente frammentazione, fornirebbe una sorta di attenuante al serrarsi in enclave di gruppi chiusi e militanti, al raggruppamento comunitario su base etno-nazionale. In particolare, le "generazioni dopo la prima" sono in Italia dei modelli di identità dinamiche e "in between" (Giorda \& Hejazi, 2019). Altri fattori (Guolo, 2019), sarebbero ancora la natura multiforme dell'associazionismo islamico, sfaccettato e a geometrie variabili, a livello sia locale sia nazionale, nonché il buon funzionamento degli apparati investigativi e dell'intelligence (Berardinelli \& Guglieminetti, 2018; Alicino, 2020).

La sopravvalutazione di cui parlavamo sopra è ben espressa nel caso delle carceri (Sonnini, 2019), nell'ambito scolastico (Giorgi \& Iannaccone, 2019; Giorda \& Giorgi, 2019, sull'invenzione mitologica delle scuole islamiche) e di recente è stata empiricamente confermata dalla difficoltà, a confronto con altri paesi europei, nel trovare individui a "rischio di" o che avessero intrapreso percorsi di radicalizzazione di natura religiosa e in particolare islamica, per lo svolgimento di un progetto sperimentale di de-radicalizzazione coordinato da uno degli autori. Tale sopravvalutazione, sebbene non basata su dati oggettivi, alimenta la paura per l'altro.

\section{Una breve storia di lunga durata e attualità della paura}

Ricostruire la storia della paura, in termini di psicologia collettiva non è l'obiettivo di questo articolo, ma possiamo riferire alcuni temi e alcune tappe di sviluppi contemporanei di una paura sociale e di una geopolitica della paura (Graziano, 2021), in particolare riferita all'Italia.

Il dubbio che può esser sollevato è che l'impiego sempre più frequente di categorie quali quelle descritte possa essere funzionale ad alimentare uno stato generico di allerta, diffuso nella società, al contempo pericoloso e utile. Pericoloso per le conseguenze etico-sociali che può comportare, utile per la 
semplificazione del messaggio e per la sua veicolarizzazione, messaggio teso ad alimentare un clima di percezione continua di minacce esterne ed interne generanti un diffuso sentimento di paura (si pensi a Todorov, 2009) finisca per prevalere su altri sentimenti sociali diffusi, a pregiudizio di politiche costruttive e condivise. Todorov (2009) ci offre con sapienza un quadro di contrapposizione valoriale e di raffigurazioni identitarie che risale diacronicamente nei secoli e ci fa comprendere come non si tratti di una dinamica meramente attuale. Sappiamo dagli studi di Delumeau (1995) sulla paura in Occidente come questa sia sempre stata una categoria presente nelle società occidentali, anche se nella sua analisi i termini, a livello sia descrittivo sia concettuale, erano riferiti a un piano trascendente, legati alla dimensione dell'ignoto e del religioso, nonché a quello della colpa e del peccato.

La proposta di Todorov (2009) in realtà si spinge oltre, collocandosi in un quadro globale geopolitico dagli equilibri stravolti - altro elemento di insicurezza e di assenza di riferimenti che acuisce il senso di incertezza diffuso - non più articolato nella distinzione tra "Oriente e Occidente" o tra "Nord e Sud", ma tra paesi "dominati dal risentimento" e paesi "dominati dalla paura", prevedendo quindi anche un rafforzamento di fenomeni populistici, nazionalistici e xenofobi. Todorov (2009) sottolinea come l'Europa sia oggi in preda alla paura nei confronti dell'Islam, paura che può rischiare di innescare reazioni violente, provocando un duplice paradosso: per un verso "la paura dei barbari rischia di trasformare noi stessi in barbari"; per l'altro "rende il nostro avversario più forte e noi più deboli".

La paura è una lente attraverso la quale si può leggere diacronicamente il processo storico e la nostra società attuale. Se, come afferma Prosperi (2021), "tremare è umano" e una certa dose di paura connota la nostra specie lungo i secoli della nostra storia, non necessariamente la risposta ai timori individuali e collettivi deve essere fondata in una direzione univoca. Egli ritrae le paure dell'uomo, soprattutto emerse nelle epoche di pestilenza, e associa la paura dell'ignoto e del trascendente al timore del male e del non poterlo controllare, spostando i timori collettivi sul piano escatologico ed etico. Del resto, esiste una vastissima iconografia "del peccato e del Male" che inevitabilmente alimenta le costruzioni intellettuali che impieghiamo per cui comprendere la nostra vita, sociale e individuale. L'analisi di Prosperi (2021) inevitabilmente arriva a spiegarci il presente, disvelando le antiche radici emotive e culturali, non solo economiche, della nostra modernità globalizzata, segnalando quanto, a suo vedere, abbiamo realmente da temere, ovvero un orizzonte di disparità economica, impoverimento culturale, saccheggio ambientale. La pestilenza ha fatto emergere, come in ogni epoca, complottismi popolari e strategie di potenti, oltre ad attacchi al sistema scientifico come lo conosciamo, a processioni penitenziali, alla diffusione di pozioni 
miracolose e alla ricerca di capri espiatori. Il monito di Prosperi ci invita a guardare oltre e riconoscere la paura nel suo aspetto più profondo e celato di forma di dominio, veleno delle menti per il quale il solo rimedio possibile è la conoscenza.

Nella seconda metà del secolo scorso, a partire dalla crisi petrolifera degli anni Settanta (anni attraversati anche dall'insorgere dei fondamentalismi religiosi), secondo Sandel (1998) sono iniziati quegli «anxious times», anni della paura, della perdita del controllo, anni di sgomento diffuso e di ricerca di ripari e protezioni anche spirituali. Come è noto, la diffusione di un maggiore senso di precarietà e la fine della fiducia incondizionata nello sviluppo economico ha contribuito, nel cosiddetto mondo occidentale e sicuramente in Italia, a rivitalizzare la ricerca spirituale e rafforzare i gruppi religiosi, bloccando il processo di secolarizzazione in corso e la decretata "morte di Dio" (Bruce, 2002). Sono stati anni cruciali per il "successo della paura": come ricorda Gardner (2008), molti sociologi individuano in quel periodo l'inizio dell'ossessione per il rischio, per la sicurezza, per la precauzione. Nel terzo millennio, con l'annus horribilis della crisi del 2008, l'«anxiety» di Sandel "si è trasformata nello Zeitgeist della nostra epoca" (Graziano, 2021, p. 10), con una moltiplicazione di paure che si intrecciano, auto-alimentano, influenzano (Moïsi, 2009).

Dal punto di vista della riflessione scientifica, in una intervista del luglio 2019, Furedi ha ricordato, a riguardo della cultura della paura: "In molti pensano che significhi che oggi le persone sono più spaventate che in passato, ma non è così. Anche perché non esiste un modo per misurare se il livello di paura di una società sia aumentato o diminuito rispetto a un dato periodo di tempo. Quello che intendo, invece, è che rispetto a qualche anno fa parliamo molto di più della paura. Sembra che tutto nella vita si associ a una preoccupazione, che qualsiasi cosa rappresenti una minaccia. L'esperienza umana in sé è ormai vista come una fonte di minaccia, anche in aree che in passato venivano viste come normali" 1 .

Sempre Furedi nel 2019 osserva: "Qualche anno fa ho condotto un progetto di ricerca che indagava le principali paure dei cittadini europei: quello che è emerso è che non erano spaventati dalle grandi questioni di cui si parlava sui giornali - come il terrorismo o il riscaldamento globale - ma da cose come l'insicurezza economica, la disoccupazione, le pensioni, i figli. L'unica cosa rilevante da un punto di vista mediatico era la paura della criminalità, per il resto si trattava di cose relativamente banali. Penso che, per quanto riguarda i cambiamenti climatici, il tema sia stato politicizzato a tal punto da

1 https://forward.recentiprogressi.it/it/rivista/numero-14-paura-coraggio/interviste/arteficidel-nostro-destino/. 
far sviluppare nei suoi confronti un'attitudine ritualistica, per cui la gente non lo prende seriamente" .

In Italia, nel 2018 il primo Rapporto sulla filiera della sicurezza di Censis e Federsicurezza aveva rilevato che "La criminalità continua ad essere ritenuta un problema grave, segnalato dal $21,5 \%$ degli italiani, al quarto posto dopo la mancanza di lavoro, indicata dal $52,4 \%$ della popolazione, l'evasione fiscale $(29,2 \%)$ e l'eccessivo prelievo fiscale". Nel 2021, nel secondo Rapporto, si legge come la paura più diffusa, per ovvie ragioni, era legata alla frequentazione di luoghi affollati, ai mezzi pubblici, ai luoghi di possibile contagio. Nessuna traccia di attentati, attacchi, terrorismo jihadista, radicalizzazioni.

Eppure, la paura per l'Islam e i musulmani è diffusa, l'islamofobia esiste, a livello istituzionale, mediatico e nella vita quotidiana. Come si legge in Islamofobia in Italia. Rapporto nazionale 2018 della Fondazione SETA, «il clima xenofobo e anti-Islam alimentato dai tradizionali attori politici della destra, Lega Nord e Fratelli d'Italia, dei movimenti di estrema destra (Casa Pound a Forza Nuova) e dai settori più conservatori dei mass-media, come ad esempio Il Giornale, ha avuto effetti molto negativi a livello sociale legittimando comportamenti di stampo razzista. Si sono accresciuti sia al Nord che al Sud gli attacchi fisici e verbali nei confronti dei migranti, richiedenti asilo, rifugiati e cittadini musulmani fino ad arrivare ad eventi drammatici» ${ }^{3}$.

Queste osservazioni, oltre a riprendere studi precedenti (si veda ad es. Pföstl, 2011; Proglio, 2020), sono state confermate anche da un recente sondaggio (Pew Research Center, 2019 citato da Lipori, 2020), che vede l'Italia all'ultimo posto tra i Paesi europei in cui si accetterebbe un musulmano in famiglia o come vicino di casa.

Ciò non stupisce e siamo, in simil compagnia: dall'indagine dell'Eurobarometro sull'integrazione degli immigrati nell'Unione europea (n. 469) emerge che la maggior parte degli europei tende a sovrastimare la quota di immigrati residente nei loro Paesi. In particolare, gli italiani stimano che la quota della popolazione immigrata sia pari a circa tre volte e mezza la cifra reale (a fronte di una media europea di tale rapporto di 2,3) (EU, 2018).

\section{Migrante, identità, radici: il potere delle parole}

La paura nei confronti degli immigrati e, in particolare, verso i mussulmani (o comunque verso chi appartiene a una religione altra rispetto a quella

\footnotetext{
$2 \mathrm{https}$ //forward.recentiprogressi.it/it/rivista/numero-14-paura-coraggio/interviste/arteficidel-nostro-destino/.

${ }^{3}$ https://confronti.net/2020/08/islamofobia-in-europa-e-in-italia/.
} 
di maggioranza) è stata alimentata negli ultimi anni da diversi soggetti. Le responsabilità sono molteplici: il mondo dei media e la sua necessità di inseguire il sensazionalismo e di individuare delle opposte tifoserie per aumentare l'audience, il mondo politico orientato al facile consenso e all'inseguimento dei sondaggi e degli umori (a volte presunti) dell'opinione pubblica senza avere il coraggio di provare ad orientarli, il mondo degli intellettuali quasi del tutto assente e afasico ma anche socialmente delegittimato dopo anni di irresponsabile elogio dell'incompetenza.

I risultati sono sotto gli occhi di tutti: l'affermarsi di un atteggiamento xenofobo diffuso che sempre più spesso sfocia nel razzismo esplicito (Barbujani, 2006; Aime, 2016; Manconi \& Resta, 2017; Lorenzini \& Cardellini, 2018; Vaccarelli, 2015, 2018), l'incapacità di interpretare i problemi e le questioni nella loro complessità e con uno sguardo lungo (Ceruti, 2018), le retoriche sovraniste delle piccole patrie sempre più radicate in molte parti del mondo, l'incapacità di pensare ad un progetto di società che contemperi solidarietà, partecipazione, sviluppo, coesione sociale e democrazia.

Il linguaggio, le parole e le categorie di analisi per affrontare questi temi ne sono usciti depauperati e impoveriti. Le parole, come ha ricordato Nanni Moretti, sono importanti perché chi parla male pensa male e vanno, perciò, maneggiate con cura. Le retoriche sovraniste hanno insistito molto in questi anni su termini quali origini, radici, identità, cultura, tradizione, ecc. Chi non ha sentito nei dibattiti pubblici o anche nei luoghi della quotidianità espressioni quali: "dobbiamo essere orgogliosi delle nostre origini, riscoprire le nostre radici, salvaguardare la nostra identità e la nostra cultura, difendere le nostre tradizioni". Affermazioni apparentemente condivisibili e di buon senso che riemergono con sempre maggior frequenza ma che dietro un velo di malcelato orgoglio nazionale spesso nascondono ben altro. Si tratta, in realtà, di parole, espressioni, nozioni, concetti che possono essere anche molto pericolosi soprattutto quando vengono piegati a logiche di esclusione e di odio verso l'altro (Zuppi \& Fazzini, 2019) falsificando la storia, manipolando gli eventi ed essenzializzando elementi dinamici e per loro stessa natura fluidi.

Il grande storico Marc Bloch, opportunamente richiamato da Montanari (2019), esprimeva già molti anni orsono tutta la sua preoccupazione per l'ambiguità del termine "origini".

«Origini» significa semplicemente «inizi»? Sarà quasi chiara allora; però con la restrizione che, per la maggior parte delle realtà storiche, il concetto stesso di questo momento iniziale rimane singolarmente evanescente. Problema di definizione, senza dubbio; ma di una definizione che, purtroppo, ci si dimentica con eccessiva facilità di dare. 
Per «origini» si dovrà intendere le cause? Non ci saranno più altre difficoltà, allora, se non quelle che costantemente (e in modo particolare, non c'è dubbio, nelle scienze umane) sono inerenti, per natura, alle ricerche causali.

Di frequente, però, tra i due significati avviene una contaminazione, tanto più temibile in quanto, in generale, poco chiaramente avvertita. Nel vocabolario comune, le «Origini» sono un inizio che spiega. Peggio ancora: che è sufficiente a spiegare. Qui sta l'ambiguità, e il pericolo (Bloch, 1969, p, 44).

Il problema è esattamente quello dell'ambiguità e dunque della conseguente pericolosità. Lo stesso dicasi in merito al tanto richiamato tema delle "radici". Lo evidenzia molto bene un altro storico come Giardina (2017) dicendo che si tratta di una metafora razzista:

la razza è paragonata ad un albero; essa non muta. Le radici della razza sono sempre le stesse. Ci sono i rami dell'albero, c'è il suo fogliame. E questo è tutto». Le buone intenzioni dei molti che oggi si appellano alle radici dei popoli, delle federazioni, delle nazioni non mutano l'essenza del problema. Anche qualora riuscissimo a confinare le risonanze di quella metafora in un ambito puramente umanistico, finiremmo sempre per constatare la sua essenza fuorviante: costruendo una gerarchia degli oggetti storici, separando i rami verdi da quelli secchi, togliendo valore creativo alle esperienze fallite o esaurite, si precipita infatti in una sorta di eugenetica storiografica (pp. XVI-XVII).

Tutta la pericolosità di questi termini è stata affrontata lucidamente da Bettini (2011) dove vengono messi in luce i limiti e, soprattutto, gli usi strumentali e demagogici di tali termini. La metafora delle radici evoca una serie di elementi, che finiscono per costituire la base di ideologie esclusiviste. Primo perché, se presa letteralmente, ci dice che noi non potremmo essere altrimenti da ciò che siamo, che la nostra cultura e la nostra identità sono segnate fin dalla nascita. Inoltre, paragonata alla radice, qualsiasi tradizione diventa fondamentale, anche dal punto di vista biologico, rispetto agli individui, non se ne può fare a meno, pena la morte. Altro punto debole dell'immagine delle radici è che la tradizione viene appresa, non ereditata geneticamente, né trasmessa attraverso la linfa o il sangue e come ogni cosa appresa, necessita di essere tenuta viva di generazione in generazione, subendo anche delle modifiche. Modifiche dovute ai cambiamenti storici e sociali e alle scelte che gli individui possono fare.

Sul tema dell'identità la letteratura è sterminata e molti studiosi (Aime, 2013; Maalouf, 1999; Prosperi, 2016; Remotti, 2010; Todorov, 2009) ci hanno insegnato a diffidare di questo termine mettendone in luce la pericolosità: "ciascuno di noi dovrebbe essere incoraggiato ad assumere la propria diversità, a concepire la propria identità come la somma delle sue diverse 
appartenenze, invece di confonderla con una sola, eretta ad appartenenza suprema e a strumento di esclusione, talvolta a strumento di guerra" (Maalouf, 1999).

È allora necessario favorire nel paese una riflessione critica sulla nozione di identità culturale, sulla pluralità delle nostre appartenenze, sulla problematicità delle culture e delle storie nazionali, sulla pericolosità dell'invenzione delle tradizioni. «Le "tradizioni" che ci appaiono, o si pretendono, antiche hanno spesso un'origine piuttosto recente, e talvolta sono inventate di sana pianta» (Hobsbawm \& Ranger, 2002, p. 19). Tale riflessione deve iniziare ed essere portata avanti nei contesti educativi e formativi.

\section{Quali modelli educativi? L'educazione interculturale}

In chiave educativa può essere utile ripartire dalle importanti considerazioni formulate da Jacques Delors nel volume Nell'educazione un tesoro. Rapporto all'UNESCO della Commissione Internazionale sull'Educazione per il Ventunesimo Secolo, pubblicato in traduzione italiana nel 1997. L'elemento principale che emerge dal rapporto della Commissione Delors è che il nostro modello di sviluppo è un modello di crescita economica che si rivela non più sostenibile rispetto a due elementi: i rapporti dell'uomo con l'ambiente e le risorse naturali; i rapporti di esclusione sociale - non solo a livello internazionale, tra nord e sud del mondo, ma anche all'interno dei singoli stati. Viene segnalata, dunque, la necessità di operare una svolta e di optare per un modello di sviluppo sostenibile (nuovi rapporti fra l'uomo e l'ambiente in cui vive e fra le persone). L'educazione, secondo il rapporto, dovrebbe favorire il passaggio dalla "crescita economica" ad uno "sviluppo umano". Oltre a suggerire una discontinuità con il modello di sviluppo dominante, il rapporto della proponeva di prendere in considerazione alcuni cambiamenti nell'ambito dei modelli educativi, che devono poter rispondere ad alcune sfide, ad alcune tensioni principali che caratterizzano il mondo contemporaneo:

- la tensione tra il globale e il locale: gli uomini debbono diventare cittadini del mondo senza perdere le loro "storie" e continuando a svolgere una parte attiva nella vita della loro nazione e della loro comunità locale;

- la tensione fra universale e particolare e la globalizzazione della cultura;

- la tensione fra tradizione e modernità, in relazione anche all'innovazione in ambito scientifico e tecnologico.

Si tratta di tensioni che richiedono all'educazione un modello sociale di riferimento in cui il passaggio fondamentale è quello dall'idea di coesione sociale all'idea e alle condizioni per un'effettiva partecipazione democratica 
per rispondere alle sfide della globalizzazione, cioè al passaggio da comunità locale a società mondiale, con aspetti dell'interdipendenza che riguardano l'ambiente, la pluralità linguistica, le migrazioni.

Un processo educativo che incoraggi la piena cittadinanza e la partecipazione democratica dovrebbe, dunque, comprendere almeno tre aree di attenzione principali:

- il rapporto locale-globale (l'idea dell'interdipendenza planetaria e delle relazioni, reti, organismi internazionali);

- la percezione dei processi di esclusione all'interno dell'attuale modello di sviluppo, che genera conflitti sociali e democrazie, dove esistono, in serio pericolo;

- l'intercultura, che sottende ad almeno quattro obiettivi pedagogici: favorire la comprensione reciproca; sviluppare il senso di responsabilità; incoraggiare la solidarietà; realizzare le condizioni per accettare le differenze spirituali e culturali.

La Commissione Delors, come è noto, ha identificato quattro pilastri dell'educazione:

- imparare a conoscere, conciliando una cultura generale sufficientemente vasta (il "passaporto per l'educazione permanente") con lo studio approfondito di un numero ristretto di materie;

- imparare a fare, sottolineando il passaggio dal concetto di abilità a quello di competenze e la possibilità di alternare scuola e lavoro;

- imparare ad essere, richiamando l'attualità delle raccomandazioni contenute nel Rapporto Faure (Unesco, 1972);

- imparare a vivere insieme, imparare a vivere con gli altri: la vera novità del rapporto, che sottolinea l'importanza di sviluppare la conoscenza degli altri popoli, della loro storia, delle tradizioni e della loro spiritualità e a partire da ciò, creare una nuova mentalità che, grazie alla consapevolezza dell'interdipendenza crescente e all'analisi condivisa dei rischi e delle sfide per il futuro, stimoli la realizzazione di progetti comuni e una gestione intelligente e pacifica degli inevitabili conflitti. È un'utopia, secondo alcuni, ma pur sempre un'utopia necessaria, un'utopia vitale per uscire dal ciclo pericoloso che stiamo vivendo fomentato dal cinismo, dalla rassegnazione, dal rifiorire della xenofobia e del razzismo.

L'educazione si pone dunque il problema di come si impara a vivere insieme, di come favorire la capacità di "mettersi nei panni degli altri”, di come imparare a progettare insieme, prevenire e trasformare i conflitti elaborando una cultura dell'accoglienza (Grassi, 2019) e della convivenza. Tali raccomandazioni appaiono oggi ancora più rilevanti di fronte ad un mondo globale che rende le nostre società sempre più multiculturali, eterogenee e ad alta complessità socioculturale (Zoletto, 2019). Di fronte a questo dato di fatto 
emergono oggi i limiti e le criticità dei principali modelli di inserimento dei migranti adottati dai diversi paesi che oscillano quasi sempre e nel migliore dei casi tra gli angusti limiti dell'assimilazionismo e del multiculturalismo. Un'altra strada è quella dell'intercultura che alcuni considerano una via desueta e che invece si vuole riproporre con convinzione perché non è mai stata percorsa seriamente e fino in fondo.

$\mathrm{Nel}$ contesto dell'Unione Europea, le differenti strategie per promuovere un approccio di educazione interculturale sono state raggruppate dalla rete europea Eurydice in diverse tipologie tra cui vi è la promozione di processi attraverso i quali le relazioni tra persone di differenti provenienze culturali vengono analizzate e rese esplicite nei curricula scolastici, con un intento interculturale che si configura a tre livelli: a) l'apprendimento di valori di rispetto e, in alcuni casi, di antirazzismo, nel quadro del contesto di diversità culturale; b) la dimensione internazionale, con un approfondimento della diversità culturale contemporanea nei contesti storici e sociali; c) l'aspetto dell'integrazione europea (Eurydice, 2004, 2009).

Una prospettiva interculturale può essere adottata non solo nella scuola dell'obbligo, ma anche nella formazione universitaria e nella formazione continua, come avvenuto nelle attività previste dal Progetto PriMed.

\section{Educare a non avere paura, prevenire le discriminazioni. Il pro- getto PriMed}

Le tematiche affrontate portano a considerare l'importanza dell'educazione alla riduzione della paura del diverso e del linguaggio dell'odio, e la decostruzione degli stereotipi e dei pregiudizi. Come ha messo in luce tra gli altri Race (2018), bisogna ridurre i pregiudizi e gli stereotipi attraverso il dialogo e l'educazione, strumenti essenziali per affrontare la complessità delle società plurali (multiculturali e multireligiose). La riduzione dei pregiudizi e degli stereotipi passa dalla conoscenza delle culture e delle religioni "diverse" e dalla capacità di "mettersi nei panni dell'altro", di cogliere il punto di vista del diverso, che si sviluppa attraverso il contatto con l'altro, sia esso diretto che indiretto (Allport, 1954; Hewestone \& Voci, 2009; Hodson \& Hewstone, 2013), come ha messo in luce anche la Commissione Jo Cox sull'intolleranza, la xenofobia, il razzismo e i fenomeni di odio della Camera dei deputati (2017). Lo sviluppo degli stereotipi e dei pregiudizi avviene sin dall'infanzia, rafforzandosi con l'età ed essendo influenzato dai media, dalla famiglia e dai coetanei (Bigler, 1999; Brown, 2010; Dovidio, Hewstone, Glick, \& Esses, 2010). Per questo la scuola e le altre istituzioni formative sono considerati i contesti privilegiati per decostruire pregiudizi e 
stereotipi e per promuovere la cittadinanza attiva e democratica (Gabrielli et al., 2019; Fiorucci, 2015). Vari studi a livello internazionale hanno verificato l'efficacia degli interventi scolastici nel ridurre gli atteggiamenti discriminatori nei confronti del diverso (tra i più recenti, Beelmann \& Heinemann, 2014; Ulger, Dette-Hagenmeyera, Reichle \& Gaertner, 2018) evidenziando come le strategie più efficaci siano proprio il contatto e la presa di prospettiva altrui o capacità di mettersi nei panni dell'altro.

$\grave{E}$ in questa prospettiva che sono stati realizzati i percorsi formativi promossi dall'unità dell'Università Roma Tre nell'ambito del progetto PriMedPrevenzione e interazione nello spazio trans-mediterraneo, finanziato dal Ministero dell'istruzione, dell'università e della ricerca nel 2019 per la prevenzione della radicalizzazione religiosa. Tale progetto coinvolge una rete di 12 università italiane e 10 dei paesi aderenti alla Organizzazione della Cooperazione Islamica (OCI). L'obiettivo è rispondere in modo interdisciplinare ai bisogni conoscitivi e operativi connessi ai processi di integrazione in Italia e al contrasto della radicalizzazione attraverso la cooperazione scientifica e la formazione di giovani e operatori.

Il lavoro dell'unità di Roma Tre è partito innanzitutto dal confronto e dal dialogo interdisciplinare tra i membri coinvolti (appartenenti a diverse discipline: storiche, pedagogiche e psicologiche) sui temi del progetto, e si è concretizzato nella sperimentazione di due corsi di alta formazione per docenti e dirigenti della scuola, e per personale della pubblica amministrazione; un laboratorio per studenti universitari sui temi della interazione tra diversità, convivenza pacifica negli spazi pubblici e mediazione; un viaggio studi a Roma che ha coinvolto circa 50 studenti di cui metà provenienti dalle università OCI coinvolte nel progetto; e da una Fall school dedicata alle narrazioni sul Mediterraneo, partecipata da studenti provenienti da tutti i paesi che si affacciano su questo mare ${ }^{4}$

In particolare il viaggio studi, coinvolgendo studenti di diverse realtà culturali e religiose, ha costituito un viaggio nei luoghi religiosi di culto presenti nello spazio urbano "super-diverso" di Roma con un continuo confronto e scambio sulle differenti realtà ed esperienze. Anche il tema della "paura" è stato centrale nelle attività formative proposte, soprattutto nel laboratorio intitolato "Paura del diverso, paura del possibile" organizzato nell'anno 2021. L'obiettivo del laboratorio è stato quello di introdurre studentesse e studenti alla storia delle rappresentazioni occidentali dell'Islam, anche ponendole in comparazione con altre tradizioni religiose. Il tema della paura dell'Islam è certamente attuale, ma ad esso si sono affiancate la tematica della paura

\footnotetext{
${ }^{4}$ Tutti le attività che si sono svolte all'interno del PriMed sono documentate nel sito: www.primed.miur.it.
} 
dentro l'Islam anche guardando alla possibile relazione causale tra suddette rappresentazioni islamofobiche e le traiettorie di radicalizzazione di musulmani in Europa e nei Paesi a maggioranza musulmana.

È infatti nostra convinzione, come abbiamo argomentato anche sulla base di quanto evidenziato dalla letteratura dei vari ambiti disciplinari, che la paura dell'altro e del diverso sia alla base delle diverse forme di radicalizzazione e che l'educazione, nelle sue diverse modalità - formale, informale $\mathrm{e}$ non formale - sia la chiave per confrontarsi con tale paura, scardinarla e permettere il dialogo alla base di una società plurale.

\section{Riferimenti bibliografici}

Aime, M. (2013). Cultura. Torino: Bollati Boringhieri.

Aime, M. (a cura di) (2016). Contro il razzismo. Quattro ragionamenti. Torino: Einaudi.

Alicino, F. (2020). Terrorismo di ispirazione religiosa. Prevenzione e deradicalizzazione nello Stato laico. Roma: Apes.

Allport, G.W. (1954). The Nature of Prejudice. Cambridge, MA: Addison-Wesley.

Antonelli, F. (2021). Radicalizzazione. Milano: Mondadori.

Barbujani, G. (2006). L'invenzione delle razze. Capire la biodiversità umana. Milano: Bompiani.

Beelmann, A., \& Heinemann, K. S. (2014). Preventing prejudice and improving intergroup attitudes: A meta-analysis of child and adolescent training programs. Journal of Applied Developmental Psychology, 31(1), pp. 10-24.

Berardinelli, D., \& Guglielminetti, L. (2018). Preventing Violent Radicalisation: The Italian Case Paradox 28. International Conference Multidisciplinary Perspectives in the quasi-coercive treatment of offenders (Specto), 7th Edition Groups With Special Needs In Community Measures. Bologna: Filodiretto pp. 20-33.

Bettini, M. (2011). Contro le radici. Tradizione, identità, memoria. Bologna: il Mulino.

Bigler, R. S. (1999). The use of multicultural curricula and materials to counter racism in children. Journal of Social Issues, 55, pp. 687-705.

Bloch, M. (1969). Apologia della storia o mestiere di storico. Torino: Einaudi.

Bombardieri, M., Giorda, M. C., \& Hejazi, S., (Eds.) (2018), Capire l'islam. Brescia: Morcelliana.

Borum, R. (2001). Radicalization into Violent Extremism. A Review of Social Science Theories. Journal of Strategic Security, 4, pp. 7-36.

Brown, R. (2010). Prejudice. Its Social Psychology. Chichester: John Wiley \& Sons Ltd.

Bruce, S. (2002). God is dead. Secularization in the West. Oxford: Blackwell Publishers. 
Camera dei Deputati - XVII legislatura (2017). Commissione Jo Cox sull'intolleranza, la xenofobia, il razzismo e i fenomeni di odio. Testo disponibile al sito: https://www.camera.it/leg17/1264 (ultima consultazione: 13/07/2021).

Ceruti, M. (2018). Il tempo della complessità. Milano: Raffaello Cortina.

Delors, J. (1997). Nell'educazione un tesoro. Rapporto all'UNESCO della Commissione Internazionale sull'Educazione per il Ventunesimo Secolo. Roma: Armando.

Delumeau, J. (1995). La paura in Occidente. Storia della paura nell'età moderna. Bologna: il Mulino.

Dovidio, J.F., Hewstone, M., Glick, P., \& Esses, V. M. (Eds.) (2010). The SAGE Handbook of Prejudice, Stereotyping and Discrimination. London: Sage Publications Ltd.

EU (2018). Special Eurobarometer 469: Integration of immigrants in the European Union, testo disponibile al sito: https://europa.eu/eurobarometer/surveys/detail/2169 (ultima consultazione: 26/05/2021).

Eurydice (2004). L'integrazione scolastica dei bambini immigrati in Europa. Brussels.

Eurydice (2009). L'integrazione scolastica dei bambini immigrati in Europa. Misure per favorire: la comunicazione con le famiglie immigrate; l'insegnamento della lingua d'origine dei bambini immigrati. Brussels.

Fiorucci, M. (2015). The Italian Way for Intercultural Education. In M. Catarci, \& M. Fiorucci, (Eds.). Intercultural Education in the European Context: theories, experiences, challenges. London, New York: Routledge.

Furedi, F. (1997). Culture of Fear: Risk-Taking and the Morality of Low Expectation. London: Cassell.

Gabrielli, S., Szpunar, G., Benvenuto, G., Maricchiolo, F., Catalano, M.G., \& Perucchini, P. (2019). Riconoscere l'Altro a scuola: il caso dei migranti. QTIMES, XI(3), pp. 86-99.

Gardner, D. (2008). The Science of Fear. New York: Dutton.

Giardina, A. (a cura di) (2017), Storia mondiale dell'Italia. Roma-Bari: Laterza.

Giorda, M. C. (a cura di) (2012). Dio lo vuole! I fondamentalismi religiosi. Torino: Sei.

Giorda, M. C, \& Giorgi, A. (2019). Religious Minorities and Faith-Based Schools in a Quasi-Religious Monopoly - the Difficulties of Inclusion. Religion \& Education, 46(2) (special section: Faith-based Schools and Religious Diversity), pp. 159-175.

Giorda, M. C, \& Hejazi, S. (2019). In between. Giovani musulmani ad eventi pubblici: il caso di Torino. Protestantesimo, 74(3-4), pp. 235-248.

Giorgi, A., \& Iannaccone, B. (2019). I giovani e la scuola: ci si radicalizza a scuola? Un'analisi dei rischi e della prevenzione della radicalizzazione in ambiente scolastico. In Bombardieri, M., Giorda, M. C., \& Hejazi, S. (Eds.). Capire l'islam. Brescia: Morcelliana, pp. 209-236.

Grassi, T. (a cura di) (2019). L'accoglienza delle persone migranti. Modelli di incontro e di socializzazione. L'Aquila: One Group.

Graziano, M. (2021). Geopolitica della paura, Milano: EGEA Boccini. 
Guolo, R. (2019). Religione e radicalizzazione. In M. Bombardieri, M. C. Giorda, \& S. Hejazi (Eds.). Capire l'islam. Brescia: Morcelliana, pp. 37-51.

Guolo, R., \& Pace, E. (1998). I fondamentalismi. Bari: Laterza.

Hewestone, M., \& Voci, A. (2009). Diversità e integrazione: il ruolo del contatto intergruppi nei processi di riduzione del pregiudizio e risoluzione dei conflitti. Psicologia sociale, 1, pp. 9-28.

Hobsbawm, E.J., \& Ranger, T. (a cura di) (2002). L'invenzione della tradizione. Torino: Einaudi.

Hodson, G., \& Hewstone, M. (2013). Advances in intergroup contact. New York, NY: Psychology Press.

Khosrokhavar, F. (2021). Jihadism in Europe: European Youth and the New Caliphate. Published to Oxford Scholarship Online: June. DOI: 10.1093/oso/9780197564967.001.0001.

Khosrokhavar, F. (2016). Prisons de France. Paris: Robert Laffont.

Khosrokhavar, F. (2014). Radicalisation. Paris: Edition Maison des Sciences de l'Homme.

Khosrokhavar, F. (2002). Les nouveaux martyrs d'Allah. Paris: Flammarion.

Lipori, M. (2020). Islamofobia in Europa e in Italia. Confronti, 26/08/2020. Testo disponibile al sito: https://confronti.net/2020/08/islamofobia-in-europa-e-in-italia/ (ultima consultazione: 13/07/2021).

Lorenzini, S., \& Cardellini, M. (a cura di) (2018). Discriminazioni tra genere e colore. Un'analisi critica per l'impegno interculturale e antirazzista. Milano: FrancoAngeli.

Maalouf, A. (1999). L'identità. Milano: Bompiani.

Manconi, L., \& Resta, F. (2017). Non sono razzista, ma. La xenofobia degli italiani e gli imprenditori politici della paura. Milano: Feltrinelli.

Marone, F., \& Vidino, L. (2018), Destinazione Jihad: i foreign fighters d'Italia. Milano: ISPI, Ledizioni LediPublishing.

Ministero dell'Istruzione-MI (2020). Gli alunni con cittadinanza non italiana. A.S. 2018/2019, Roma. Testo accessibile al sito: https:/www.miur.gov.it/documents/20182/0/Rapporto+-+Gli+alunni+con+cittadinanza+non+italiana_as_2018-2019.pdf/f1af9f21-cceb-434e-315e$5 \mathrm{~b} 5 \mathrm{a} \overline{7} \mathrm{c} 55 \mathrm{c} 5 \mathrm{db}$ ? $=1616517692793$ (ultima consultazione: 13/07/2021).

Moïsi, D. (2009). The Geopolitics of Emotion: How Cultures of Fear, Humiliation, and Hope are Reshaping the World. New York: Doubleday.

Montanari, M. (2019). Breve storia degli spaghetti al pomodoro. Roma-Bari: Laterza.

Pasquinelli, C. (1993). Fondamentalismi, 3. PAROLECHIAVE nuova serie di "Problemi del socialismo" 3/1993.

Pföstl, E. (2011). Between Fear and Integration: Islamophobia in Contemporary Italy. European Yearbook of Minority Issues, VIII(1), pp. 45-70.

Proglio, G. (a cura di) (2020). Islamofobia e razzismo. Media, discorsi pubblici e immaginario nella decostruzione dell'altro. Torino: Edizioni SEB27.

Prosperi, A. (2016). Identità. L'altra faccia della storia. Roma-Bari: Laterza. 
Prosperi, A. (2021). Tremare è umano. Una breve storia della paura. Milano: Solferino.

Race, R. (Ed.) (2018) Advancing Multicultural Dialogues in Education. Houndsmills: Palgrave Macmillan.

Remotti, F. (2010). L'ossessione identitaria. Roma-Bari: Laterza.

Sandel, M. (1998). Democracy's Discontent: America in Search of a Public Philosophy. Cambridge: Harvard University Press.

Serafini, M. (2015). Maria Giulia che divenne Fatima. Storia della donna che ha lasciato l'Italia per l'Isis. Milano: RCS Media Group.

Sonnini, E. (2019), L'islam in carcere e il mito del radicalsmo. In Bombardieri, M., Giorda, M. C., \& Hejazi, S. (Eds.). Capire l'islam. Brescia: Morcelliana, pp. 187208.

Squarcini, F., \& Tavarnesi, L. (a cura di) (2007). Fondare i fondamentalismi. Esplorazioni critiche dei diversi modi del fondamentalismo nella storia. Firenze: Società Editrice Fiorentina.

Todorov, T. (2009). La paura dei barbari. Oltre lo scontro delle civiltà. Milano: Garzanti.

Ulger, Z., Dette-Hagenmeyera, D.E., Reichle, B., \& Gaertner, S.L. (2018). Improving outgroup attitudes in schools: A meta-analytic review. Journal of School Psychology, 67, pp. 88-103. Doi: 10.1016/j.jsp.2017.10.002.

Vaccarelli, A. (2015). Razzismo. In Catarci M., \& Macinai, E. (Eds.). Le parole chiave della pedagogia interculturale. Pisa: ETS.

Vaccarelli, A. (2018). Mala tempora currunt. L'educazione interculturale come sfida e come necessità. In L. Agostinetto, M. Fiorucci, \& S. Polenghi, Diritti Cittadinanza Inclusione. Lecce: Pensa Multimedia.

Wilner, A., \& Dubouloz, C. (2010). Homegrown Terrorism and Transformative Learning; An Interdisciplinary Approach to Understanding Radicalization. Global Change, Peace and Security, 22(1), pp. 33-51.

Zoletto, D. (2019). A partire dai punti di forza. Popolar culture, eterogeneità, educazione. Milano: FrancoAngeli,

Zuppi, M. M., \& Fazzini, L. (2019). Odierai il prossimo tuo. Perché abbiamo dimenticato la fraternità. Riflessioni sulle paure del tempo presente. Milano: Piemme. 